http://jmscr.igmpublication.org/home/ ISSN (e)-2347-176x ISSN (p) 2455-0450 crossref DOI: https://dx.doi.org/10.18535/jmscr/v8i3.35

\author{
D) Journal Of Medical Science And Clinical Research

\title{
Perforated Duodenal Gist - A Rare Presentation
}

\author{
Authors \\ Aditya Sivaram $\mathbf{S}^{1}$, Mohanapriya $\mathbf{T}^{2}$, Balaji Singh $\mathbf{K}^{3}$, Manuneethi Maran \\ Dept of General Surgery, Sri Ramachandra Institute for Higher Education and Research, Chennai, India
}

\begin{abstract}
Duodenal GISTs are quite a rare phenomenon and their management is very tricky depending on the part of the duodenum from where it arises. We report a case of 68 year old male from West Bengal, India presenting with features of sealed off perforation with a retroperitoneal collection and finally diagnosed with High Grade GIST from duodenum (D1-D2), Spindle cell Type, $T_{4} N_{x} M_{0}$. Patient underwent Wedge Resection of the tumour followed by Imatinib therapy.
\end{abstract}

Keywords: GIST, Duodenal Gist, Perforation.

\section{Introduction}

Gastro Intestinal Stromal Tumours (GISTs) are most common tumour of mesenchymal origin in gastrointestinal tract. The arise from the interstitial cells of Cajal, pacemaker cells of the intestine. Most common site involved is the stomach followed by the small intestine. The presentation has been highly variable from being asymptomatic to intussusceptions and obscure bleeding. Clinical presentation as perforation of the tumour is extremely rare and makes it difficult for the diagnosis.

\section{Case Report}

A 68 year old male patient came to the hospital with complaints of abdominal pain and abdominal distension for past 15 days. Patient developed vomiting containing food particles, non bilious, not bloodstained, intermittent, associated with dull aching abdominal pain for the 2 days, the patient also had 4 episodes of malena for 2 days. The patient did not have any history of fever, constipation, loss of weight or loss of appetite. The patient was a known case of diabetes mellitus and hypertension. patient was an old case of pulmonary tuberculosis and treated completely .

On examination, patient was conscious and oriented, moderately built and nourished, general examination was normal, no signs of dehydration, vitals stable. Abdomen was soft, there was mild tenderness in epigastric and right hypochondrium, there was no guarding or rigidity of the abdomen. Other systems were normal.

Baseline investigations were done and haemoglobin 9.3, total blood count 4600 , Renal function test and liver function test were normal. Upper GI endoscopy was done which showed grade B distal esophagitis and extrinsic subepithelial swelling noted in D1. Contrast Enhanced CT of Abdomen done, which showed a lesion of size 11.4 x $17.1 \mathrm{x}$ $15.3 \mathrm{~cm}$ noted occupying the right hypochondrial and lumbar regions. Irregular walls noted, nodular enhancing irregular anteromedial, medial and posteromedial, walls appearing adherent to the second part of the duodenum with partially deficient lateral wall. Findings suggested a possibility of a exophytic GIST arising from the second part of the 


\section{JMSCR Vol||08||Issue||03||Page 214-217||March}

duodenum with central necrosis and sealed perforation from the lateral wall and potential fistulous connection with duodenum.

The patient initially underwent pig tail catheterisation of the collection. Appropriate antibiotics was started. After getting the anaesthetic fitness, The patient was posted for Exploratory Laparotomy, intra op findings were $12 \times 10 \mathrm{~cm}$ retroperitoneal mass lesion arsing from D1-D2 junction with solid and haemorrhagic components, medial wall of the duodenum was not involved, extensive adhesions noted between the lesion and liver, gall bladder, transverse colon, hepatic flexure and retro peritoneum noted. Duodenal GIST excision and Wedge Resection and Anastamosis of duodenum and Gastrojejunostomy was done. Final histopathology was reported as High Grade GIST $\mathrm{pT}_{4} \mathrm{~N}_{\mathrm{x}} \mathrm{cM}_{0}$, spindle cell type. By IHCs, tumour was positive for Vimentin, CD 117, DOG-1 and negative for SMA, S-100. Patient was treated with Adjuvant Chemotherapy with Imatinib.

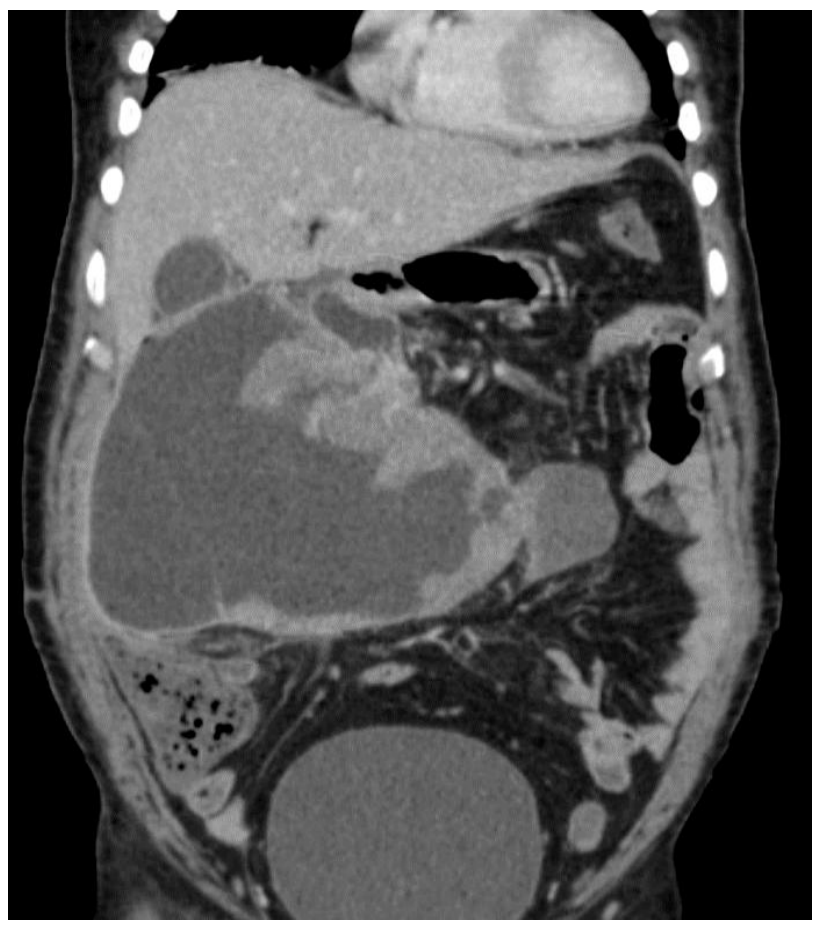

Fig. 1 Coronal Section of CT scan showing the collection and the lesion

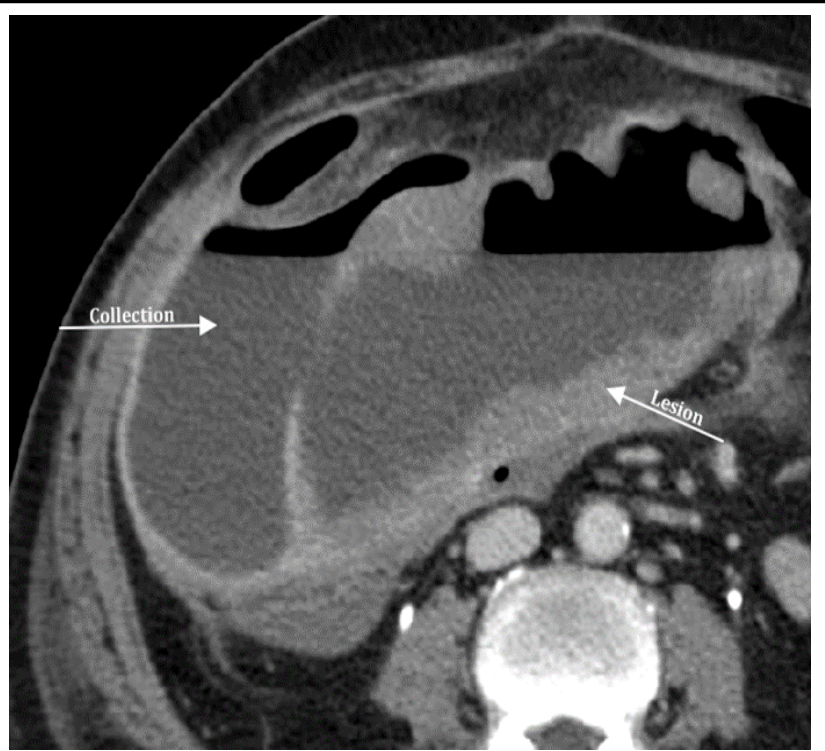

Fig 2. Axial Section - CECT Abdomen - GIST

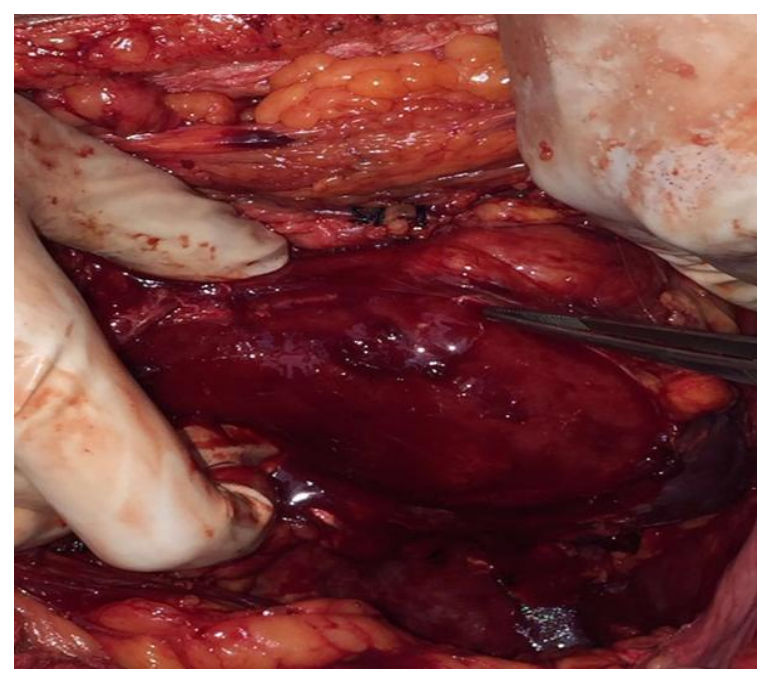

Fig 3 Perforated Site

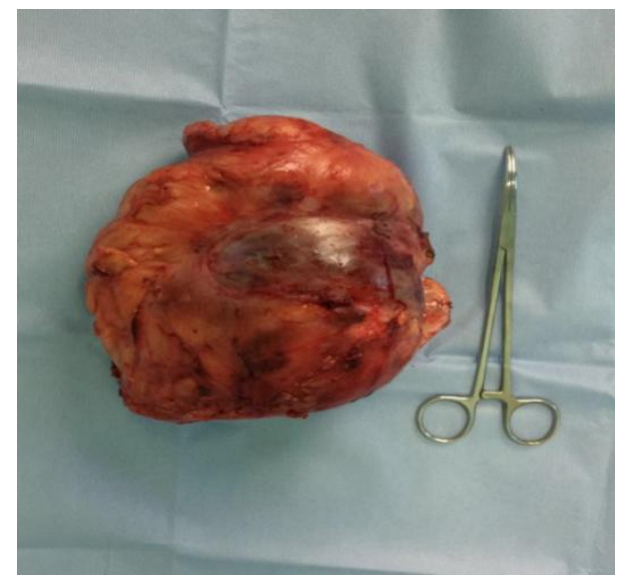

Fig. 4 GIST - Duodenum

\section{Discussion}

Gastrointestinal stromal tumours (GISTs) are the most common malignant subepithelial lesions of the gastrointestinal tract. They originate from the 
interstitial cells of Cajal located within the muscle layer and are characterized by over-expression of the tyrosine kinase receptor KIT.GISTs most commonly occur in the stomach $(51 \%)$, followed by the small intestine (36\%), colon (7\%), rectum (5\%), and oesophagus (1\%). ${ }^{1}$ Small intestine is the second most common location of GIST after stomach. Tumour can arise from throughout the small intestine, i.e. duodenum, jejunum and ileum. Tumour extending into the bowel lumen may cause intestinal obstruction while those with extra-serosal extension rarely cause intestinal obstruction. ${ }^{2}$

The most common symptoms of GISTs are gastrointestinal bleeding, including acute melena and hematemesis with subsequent anaemia; weakness; and abdominal pain, distension, and discomfort due to a tumour-induced mass effect. ${ }^{3}$ GISTs can either be asymptomatic, found incidentally at the time of GI evaluation by either endoscopic or contrast studies, at operation, or may cause life-threatening symptoms.

Symptoms are site specific and include: dysphagia for tumours of the oesophagus, nausea and vomiting caused by gastric outlet obstruction for gastric tumours, obstructive jaundice for periampullary tumours, and bowel obstruction usually caused by intussusceptions for tumours of the small bowel and colon. In up to $20 \%$ of patients, an asymptomatic abdominal mass is the presenting complaint.

Duodenal GISTs account for only $<5 \%$ but make up $30 \%$ of primary duodenal tumors. They usually present with abdominal pain to due obstruction, anemia, or gastrointestinal bleeding from a central ulceration. Small duodenal GISTs may be incidental findings during gastroscopy. ${ }^{4}$ Grossly, GISTs typically present as a sharply demarcated mass lesion without lymphatic spread, arising in the submucosa ${ }^{[6]}$. Histologically, spindle cell $(70 \%)$, epithelioid $(20 \%)$ or mixed type differentiation can be observed, depending on tumor site. the results of mutation analysis of the KIT and PDGFRA gene and comparative genomic hybridization $(\mathrm{CGH})$ are employed as additional prognostic factors with impact on diagnosis and therapy. The diagnosis is made by CECT abdomen. Newer techniques like endoscopic ultrasound and subsequent FNA or biopsy can also be done. GISTs in the duodenum do not differ from other GISTs in immunohistochemical reaction. Most of them express CD-117 (c-kit) and CD-34. Which has been found to be associated with worse prognosis. PDGFRA expression is less frequent, and there is no relation to a different prognosis. ${ }^{5}$

Coming to the treatment part, surgical treatment remains the gold standard modality for the disease. a $\mathrm{R} 0$ resection is supposed to be associated with better outcome irrespective of the histological grading. In duodenum, both limited and extended surgery yield comparable survival rates, tumour size and location in regard to the papilla of Vater, associated diseases and the patient's performing state should be considered when deciding between segmental duodenectomy and pylorus-preserving duodeno-pancreatectomy . ${ }^{6}$

Imatinib mesylate, a tyrosine kinase inhibitor, plays a key role in the management of GISTs. Its use in neoadjuvant therapy, adjuvant therapy and in tumor recurrence has dramatically changed the natural history of metastatic and recurrent GISTs.

In the neoadjuvant setting for GISTs located in the second portion of the duodenum, Imatinib mesylate has been used for tumour down staging in order to perform a less extensive surgery with free resection margins. ${ }^{7}$ However, this requires a precise preoperative diagnosis of GIST which is not always easy to obtain. The use of Imatinib as adjuvant therapy, or for local or metastatic recurrence of duodenal GIST, should not differ from that for other GISTs, and the treatment should be continued indefinitely (usually at a dose of $400 \mathrm{mg} /$ day). Interruption of treatment is generally followed by rapid tumor progression. The major limitation of Imatinib is the development of tumor resistance, which is related to the acquisition of additional c-kit mutations. Since the failure of imatinib, sunitinib malate, a multi-target TKI with powerful activity against KIT and PDGFRA, as well as a number of other kinases, has demonstrated efficacy as a second-line therapy and is now approved globally for use in metastatic GIST in patients who are 
resistant or intolerant to imatinib. Regorafenib is an oral TKI that inhibits numerous kinases that participate in oncogenesis, angiogenesis, and the tumor microenvironment have been implicated in GISTs resistant to imatinib and sunitinib. ${ }^{8,9}$

\section{Conclusion}

Duodenal GISTs are extremely rare entity. The variable presentation makes it hard for the diagnosis. The treatment would be the same for GIST from any other site - R0 Surgical Resection. Wedge resection of the tumour can be performed wherever possible. pancreaticoduodenectomy is done in lesions near to the ampulla. Imatinib as adjunct is mandatory.

\section{References}

1. Akahoshi, Kazuya et al. "Current clinical management of gastrointestinal stromal tumor." World journal of gastroenterology vol. 24,26 (2018): 2806-2817.

2. Panbude S, Ankathi S, Ramaswamy A, Saklani A. Gastrointestinal Stromal Tumor (GIST) from esophagus to anorectum \&\#8211; diagnosis, response evaluation and surveillance on computed tomography (CT) scan. Indian J RadiolImaging. 2019 Apr 1;29(2):133-40.

3. Gastrointestinal stromal tumour. Joensuu H, Hohenberger P, Corless CL Lancet. 2013 Sep 14; 382(9896):973-83

4. Beham A, Schaefer I-M, Cameron S, von Hammerstein K, Füzesi L, Ramadori G, et al. Duodenal GIST: a single center experience. Int J Colorectal Dis 2013

5. Huang Y, Chen G, Lin L, Jin X, Kang M, Zhang Y, et al. Resection of GIST in the duodenum and proximal jejunum: A\&\#xa0; retrospective analysis of outcomes. Eur J Surg Oncol. 2019 Oct 1;45(10):1950-6.

6. Hong L, Zhang T, Lin Y, Fan R, Zhang M, Cheng $\mathrm{M}$, et al. Prognostic Analysis of Duodenal Gastrointestinal Stromal Tumors. De Palma GD, editor. Gastroenterol Res Pract .2018;2018:4812703.
7. Parab TM, De Rogatis MJ, Boaz AM, Grasso SA, Issack PS, Duarte DA, et al. Gastrointestinal stromal tumors: a comprehensive review. J Gastrointest Oncol. 2019 Feb;10(1):144-54.

8. Duffaud F, Le Cesne A. Recent advances in managing gastrointestinal stromal tumor. F1000 Research . 2017 Sep 14;6:1689.

9. Call JW, Wang Y, Montoya D, Scherzer NJ, Heinrich MC. Survival in advanced GIST has improved over time and correlates with increased access to post-imatinib tyrosine kinase inhibitors: results from Life Raft Group Registry. Clin Sarcoma Res. 